\title{
Philosophiques
}

\section{Plaider l'égalité pour mieux la dépasser : Gabrielle Suchon et l'élévation des femmes}

\section{Charlotte Sabourin}

Volume 44, numéro 2, automne 2017

Les nouveaux horizons du féminisme dans la philosophie francophone

URI : https://id.erudit.org/iderudit/1042331ar

DOI : https://doi.org/10.7202/1042331ar

Aller au sommaire du numéro

Éditeur(s)

Société de philosophie du Québec

ISSN

0316-2923 (imprimé)

1492-1391 (numérique)

Découvrir la revue

Citer cet article

Sabourin, C. (2017). Plaider l'égalité pour mieux la dépasser : Gabrielle Suchon et l'élévation des femmes. Philosophiques, 44(2), 209-232.

https://doi.org/10.7202/1042331ar
Résumé de l'article

Cet article se penche sur la contribution de Gabrielle Suchon à la célèbre "querelle des femmes ". J’y démontre que, quoique Suchon défende l'égalité des deux sexes en ce qui a trait à leurs capacités à la liberté, à la science et à l'autorité, elle vise ultimement à montrer que les femmes peuvent se rendre supérieures aux hommes. Je montrerai que son projet d'élévation des femmes ne peut être accompli qu'en soustrayant les femmes à l'influence des hommes, les relations entre les deux sexes étant foncièrement préjudiciables aux premières. 


\title{
Plaider l'égalité pour mieux la dépasser: Gabrielle Suchon et l'élévation des femmes
}

\author{
CHARLOTTE SABOURIN \\ Université McGill \\ charlotte.sabourin@mail.mcgill.ca
}

\begin{abstract}
RÉSUMÉ. - Cet article se penche sur la contribution de Gabrielle Suchon à la célèbre «querelle des femmes». J'y démontre que, quoique Suchon défende l'égalité des deux sexes en ce qui a trait à leurs capacités à la liberté, à la science et à l'autorité, elle vise ultimement à montrer que les femmes peuvent se rendre supérieures aux hommes. Je montrerai que son projet d'élévation des femmes ne peut être accompli qu'en soustrayant les femmes à l'influence des hommes, les relations entre les deux sexes étant foncièrement préjudiciables aux premières.
\end{abstract}

ABSTRACT. - This paper examines Gabrielle Suchon's contribution to the famous "querelle des femmes". I argue that although Suchon is a proponent of the equality of the sexes with respect to their capacities for freedom, science and authority, she ultimately suggests that there is a way in which women can make themselves superior to men. I will show that her project for the elevation of women can be accomplished only by isolating women from men, given that the relationships between the sexes will always be detrimental to women.

Les hommes ont tellement dégéneré de l'estime a de la tendresse qu'ils doivent aux personnes du Sexe, que l'on peut dire avec verité, qu'ils ont renoncé à une partie d'eux mémes, par les mépris qu'ils font continuellement des femmes'.

La "querelle des femmes" est une appellation très large, renvoyant à un débat portant autant sur la nature féminine et sur la différence sexuelle que sur les rapports entre hommes et femmes, et sur la place des femmes dans la société. Or, à la différence d'autres célèbres querelles circonscrites dans le temps et dans l'espace (on peut penser par exemple à la querelle du panthéisme), la querelle des femmes renvoie en fait à une pluralité de débats ayant eu cours du XIV ${ }^{\mathrm{e}}$ au XVIII ${ }^{\mathrm{e}}$ siècle, tant en France qu'en Italie, en Angleterre, en Espagne et autres nations européennes. Dans ces conditions, on comprend que, malgré certaines références communes (Platon, Aristote, Plutarque, Sénèque, etc.), chaque contribution doit être comprise dans le contexte qui lui est propre. Celle de Gabrielle Suchon s'inscrit ainsi dans la France du XVII ${ }^{\mathrm{e}}$ siècle, dans la foulée d'autres défenses des femmes comme celles de Pierre Le Moyne (La gallerie des femmes fortes, I647), Jacquette Guillaume (Les Dames illustres, où par bonnes et fortes raisons il se prouve

1. Suchon, Petit traité de la faiblesse, de la légèreté et de l'inconstance qu'on attribue aux femmes mal à propos. Préface.

PHILOSOPHIQUES 44/2 - Automne 2017, p. 209-232 
que le sexe féminin surpasse en toutes sortes de genres le sexe masculin, I665) et François Poulain de la Barre (De l'égalité des deux sexes, I673), pour n'en nommer que quelques-unes - d'ailleurs évoquées par Suchon elle-même ${ }^{2}$.

La contribution de Suchon prend la forme de deux traités volumineux, le Traité de la morale et de la politique divisé en trois parties, sçavoir, la liberté, la science et l'autorité, où l'on voit que les personnes du sexe, pour en être privées, ne laissent pas d'avoir une capacité naturelle, qui les en peut rendre participantes (ci-après: Traité de la morale et de la politique), publié en I693, et le traité $D u$ célibat volontaire, ou la vie sans engagement (ciaprès: Célibat volontaire), publié en $1700^{3}$. Tous deux participent, à mon sens, d'un seul et même projet: celui d'une élévation des femmes. Car, et c'est la thèse que je défendrai ici, Suchon ne pose l'égalité des hommes et des femmes que pour mieux la dépasser — c'est-à-dire afin de montrer que, sous certaines conditions, celles-ci peuvent devenir meilleures que ceux-là, et donc supérieures.

Il faut savoir que, étant donné son caractère éclectique, la querelle vient mobiliser une pluralité de genres littéraires ${ }^{4}$, mais également de lignes argumentatives. Face aux nombreux détracteurs du sexe féminin ${ }^{5}$, les défenseurs des femmes argumentent tantôt en faveur de leur supériorité, tantôt dans le sens d'une égalité des deux sexes. Gabrielle Suchon peut à bien des égards être rangée dans cette seconde catégorie: le Traité de la morale et de la politique vise en effet à établir hors de tout doute que les femmes possèdent les capacités nécessaires à l'exercice de la liberté, de la science et de l'autorité; et ce, au même titre que les hommes - une thèse qui, on s'en doute, n'allait pas de soi à l'époque de la rédaction du Traité. J'espère néan-

2. Suchon, Traité de la morale et de la politique, Préface générale. Chaque section du Traité de la morale et de la politique étant paginée de façon indépendante (à l'exception des préfaces, non paginées), les références seront effectuées en utilisant le thème de chaque section (soit Liberté, Science et Autorité) suivi du numéro de page de l'édition originale.

L'édition originale peut être consultée en version numérisée sur Gallica, le site de la Bibliothèque nationale de France; sa transcription intégrale sera bientôt disponible au http:// querelle.ca/. L'œuvre de Suchon a été partiellement rééditée par Séverine Auffret; je référerai malgré tout à la pagination de l'édition d'origine dans le but d'assurer l'uniformité des références.

3. Outre ces deux ouvrages, on a de bonnes raisons de croire que Suchon aurait écrit un troisième traité, malheureusement jamais publié et sans doute égaré: le Traité de l'excellence de la Vocation des filles consacrées à Dieu dans le Cloitre et de la sainteté de leurs exercices divisé en trois tomes pendant Le tems de huit années. Elle y réfère d'ailleurs dans Le célibat volontaire (40I). On peut consulter à ce sujet les recherches documentaires de Kirsop, 2005 ; et de Desnain, 2013 , p. $265-266$.

4. L'éclectisme des genres littéraires mobilisés (poèmes, harangues, essais, etc.) est sans doute en partie responsable du manque d'attention accordé à la querelle en philosophie.

5. On peut penser notamment à la Louenge des femmes d'André Misogyne (I 55 I), à l'Alphabet de l'imperfection et malice des femmes de Jacques Olivier (I 6 I 7 ) et à la Cacogynie du Sieur de Fierville (I6I7). 
moins montrer que, sur les bases de cette égalité des capacités, Suchon aspire en fait à montrer que les femmes peuvent devenir meilleures que les hommes; non pas pour les dominer, mais bien plutôt parce qu'elle considère qu'il s'agit de la meilleure façon d'améliorer la société tout entière.

Le projet de Suchon tient ainsi en deux volets, dont le premier consiste à démontrer l'égalité des capacités des hommes et des femmes en ce qui a trait à la liberté, à la science et à l'autorité. Cette première partie du projet est mise en œuvre dans le Traité de la morale et de la politique et, afin de la mener à bien, Suchon doit répondre aux nombreuses accusations d'infériorité dont les femmes font les frais. La citation suivante résume bien sa position:

La contrainte, l'ignorance, \& la dependance où les personnes du Sexe passent leur vie renferment toutes les peines qui les rendent inferieures aux hommes; de sorte que dans la Privation de Liberté, de Science, \& d'Autorité, l'on peut connoître qu'elles n'ont point de part à tous les plus grands avantages que l'on possede dans la Politique \& dans la morale ${ }^{6}$.

De fait, ces privations ont des conséquences extrêmement néfastes pour les femmes, qui «font la morale sans même la connaître ${ }^{7}$ » et qu'on éduque à peine assez pour les élever au-dessus du niveau des bêtes. L'infériorité apparente des femmes est donc le fait de coutumes regrettables, non la conséquence d'une différence sexuelle naturelle. Cependant, cette démonstration d'une égalité des capacités ne constitue qu'une partie du projet de Suchon et doit être complétée par l'élévation des femmes au-dessus du reste de l'humanité corrompue - un idéal rendu possible par la condition de neutraliste qu'elle crée dans son traité $\mathrm{Du}$ célibat volontaire.

Le deuxième volet du projet de Suchon consiste ainsi à permettre aux femmes de se perfectionner, sur la base des capacités qu'elles possèdent. Il faut noter d'emblée que cette amélioration ne profitera pas qu'aux femmes elles-mêmes, mais bien à la société entière, hommes y compris ${ }^{8}$. Avec ce projet d'élévation des femmes, Suchon poursuit en fait un triple objectif maintes fois réitéré:

Je peux dire avec vérité que tous mes desirs \& toutes les intentions que j'ay eu dans cét Ouvrage ne sont que de rendre service à Dieu, \& de procurer la réformation, l'utilité \& la perfection des personnes du Sexe'.

6. Suchon, Traité de la morale et de la politique, préface générale.

7. Suchon, Science, p. 44.

8. Cette dimension politique du projet de Suchon, relevée par Dorlin, 200 I Fauré, I985; Le Doeuff, I998; et Shapiro (à paraître), constitue le point de départ d'une étude très instructive de Desnain (2013), qui y expose les diverses stratégies argumentatives et rhétoriques utilisées par Suchon.

9. Suchon, Traité de la morale et de la politique, conclusion générale, p. I38. Voir aussi la préface générale. 
Bien que les femmes soient à la fois les exécutantes et premières bénéficiaires de ce projet ambitieux, il ne peut être séparé de ses motivations religieuses. En incitant les femmes à se perfectionner, Suchon a - évidemment - en vue leur propre bien-être; mais elle aspire également à rendre grâce à Dieu et à servir la société. Cette préoccupation religieuse est ainsi essentielle à la bonne compréhension de l'œuvre de Suchon ${ }^{10}$, puisque l'amélioration des femmes doit servir aussi bien Dieu que la société.

On verra ainsi que si Suchon défend bel et bien une égalité de capacités, elle ne peut être rangée parmi les tenants d'un égalitarisme visant à assimiler les femmes à un modèle soi-disant égalitaire et qui serait en fait pensé à partir des hommes ${ }^{11}$. Bien au contraire, pour Suchon, la vaste majorité des hommes sont corrompus par le pouvoir qu'ils détiennent. L'élévation des femmes ne peut donc être accomplie en adoptant l'homme pour modèle; elle doit plutôt viser à le dépasser. Afin de faire sens de cet ambitieux projet, je décrirai d'abord les deux volets annoncés plus haut - la démonstration de l'égalité des capacités et le projet d'amélioration des femmes -, afin de montrer en un troisième temps que la solution proposée par Suchon, soit la condition de neutraliste, ne peut reposer que sur les femmes elles-mêmes et requiert, pour leur élévation, de les soustraire définitivement au pouvoir des hommes.

\section{L'égalité des capacités}

Il convient d'abord de donner un aperçu du premier volet du projet de Suchon en montrant de quelle façon elle établit que les femmes possèdent par nature les mêmes capacités que les hommes, et que c'est plutôt la coutume qui en fait des êtres inférieurs. On verra enfin que la coutume doit elle-même être expliquée par l'intérêt bien compris des hommes, au sens sexué du terme; car ces derniers retirent des avantages — ou du moins le croient-ils — de la subordination des femmes.

\section{Suchon et les traditions argumentatives de la querelle des fermmes}

Afin d'établir hors de tout doute que les femmes sont des êtres raisonnables à part entière et, à ce titre, possèdent les capacités nécessaires à l'exercice de

10. Desnain montre bien l'importance que détenait cette dimension religieuse dans le féminisme de la modernité, dimension parfois évacuée par les commentateurs contemporains (Desnain, 2006). Au sujet des motivations religieuses de Suchon, on peut consulter les études de Bertolini, 2000; et de Ribard, 2009.

11. Ce reproche a été adressé tout particulièrement aux féministes du XviII siècle, Mary Wollstonecraft en première instance. Schiebinger résume bien cette position: "Tout en appelant à l'égalité, les égalitaires demeurent convaincus que ce sont les femmes (et pas les hommes ou la société) qui doivent être réformées. Pour les féministes de la période prérévolutionnaire, l'aspiration à partager les droits des hommes implique que les femmes doivent acquérir les compétences des hommes - et pas que les hommes doivent acquérir les compétences traditionnellement réservées aux femmes " (Schiebinger, I989, p. 230-23 I ; ma traduction). 
la liberté, de la science et de l'autorité, Suchon procède par voie de raisonnements, d'autorités et d'exemples. Elle ne se montre pas particulièrement originale à cet égard, ces trois procédés étant utilisés par la quasi-totalité des protagonistes de la querelle des femmes. Les raisonnements de Suchon visant à établir l'égalité de capacité des hommes et des femmes sont ainsi des arguments repris par nombre de tenants de l'égalité des deux sexes. Cette uniformité est également perceptible en ce qui concerne les exemples utilisés: les femmes exemplaires citées par Suchon sont bien connues et recensées dans plus d'un catalogue de femmes d'exception. Il vaut cependant la peine de s'attarder sur le recours à l'autorité, procédé très fréquent dans la querelle et qui devait valoir de sévères critiques à celles qui l'ont utilisé: on accuse ainsi volontiers Suchon et ses consœurs de manquer d'originalité en se bornant à reprendre les arguments élaborés par de grands penseurs avant elles ${ }^{12}$, ou encore d'avoir déformé les propos de ceux qu'elles citent ${ }^{13}$.

De fait, les traités de Suchon sont particulièrement remarquables par le nombre et la richesse de leurs références (bibliques, anciennes et modernes), ce dont l'auteure est bien consciente. Elle refuse ainsi d'associer ses nombreuses références à une "mendicité » ou à une "nudité d'esprit ", alléguant qu'il est, au contraire, extrêmement profitable de "suivre leurs lumières pour fortifier les nôtres et éclaircir nos difficultés ${ }^{14}$. Suchon va même jusqu'à affirmer qu'elle n'aurait jamais osé écrire sans s'assurer d'avoir l'appui des écritures saintes et de philosophes et théologiens variés, qu'elle qualifie de "conducteurs fidèles » et de "guides éclairés ${ }^{15}$. Par ailleurs, les nombreuses références, citations et paraphrases ponctuant son œuvre constituent, au final, un exercice fort intéressant de réappropriation des sources. L'auteure est d'ailleurs très consciente de prendre certaines libertés par rapport à ceux qu'elle cite, faisant de cette réappropriation un pivot de son argumentation:

Les écrits des sçavans peuvent recevoir diverses expositions, \& s'accommoder aux differentes necessitez des ames. S. Bernard parlant des écritures sacrées nous assure qu'on les peut expliquer en divers sens, sans commettre aucune absurdité. Qu'elle raison peut on avoir pour trouver mauvais que les saintes lettres, les livres des Peres, \& des Auteurs graves soient appliquez differemment pourveu que l'on suive toûjours la foy Catholique $\&$ orthodoxe; puisque les choses materielles sont propres à divers usages \& servent à plusieurs \& differentes fonctions ${ }^{16}$.

12. Voir par exemple dans l'excellent ouvrage d'Angenot: "Les femmes qui au $\mathrm{XVII}^{\mathrm{e}}$ siècle se mettent à parler de leur condition, Mesdemoiselles de Gournay, de Nervèze, Anne-Marie van Schurman, le font en reprenant et relayant les arguments mis au point par des hommes et s'éprouvent donc elles-mêmes comme le lieu d'un paradoxe.» Angenot, I977, p. 53 .

13. Pour Suchon en particulier, on pense notamment à Ronzeaud, I975.

14. Suchon, Traité de la morale et de la politique, préface générale.

15. Suchon, Traité de la morale et de la politique, préface générale.

16. Suchon, Traité de la morale et de la politique, préface générale. 
De fait, Suchon se sert d'arguments ou de portions d'arguments élaborés par certains théologiens ou philosophes lorsque cela convient à sa propre argumentation - et donc de façon sélective ${ }^{17}$. Loin de constituer un plagiat ou une déformation, il s'agit d'une récupération délibérée et pleinement assumée. Le but des défenseurs des femmes n'est pas de faire preuve d'originalité, mais plutôt de montrer que les femmes sont égales aux hommes et que cet état de choses peut être cautionné par le recours à des auteurs dont l'autorité est incontestable. Par ailleurs, comme le souligne Dorlin, affirmer l'égalité des deux sexes en faisant usage de sources jugées respectables constitue en soi un geste passablement subversif: Suchon et ses consœurs viennent défendre «des thèses hétérodoxes en s'aidant des références les plus orthodoxes ${ }^{18} »$. Suchon s'inscrit donc indéniablement dans une tradition argumentative propre à la querelle.

L'articulation du Traité de la morale et de la politique est, quant à elle, quelque peu inhabituelle pour un traité de la querelle. Suchon dévoile en effet, dès la préface, les trois causes de l'infériorité des femmes, soit la privation de liberté, la privation de science, et la privation d'autorité. Or, avant même de désigner ces notions de façon positive (liberté, science, autorité), Suchon introduit leurs pendants négatifs, soit respectivement la contrainte, l'ignorance et la dépendance. Le traité décrit méthodiquement ces trois notions, sans jamais oublier leur pendant négatif. Ainsi, dans le premier traité, la liberté fait d'abord l'objet d'une définition en bonne et due forme. Une fois l'essence de la liberté établie, ses différentes espèces et propriétés font l'objet d'une exposition méthodique dans les chapitres suivants, suivie des différentes manières de l'exercer et de ses effets. La capacité des femmes à être libre n'est réellement établie qu'à ce stade. Enfin, le pendant négatif de la liberté, soit la contrainte, fait l'objet de la seconde moitié de cette première partie. Tout comme pour la liberté, Suchon commence par définir la contrainte, avant d'exposer ses espèces, ses propriétés et ses effets. Les deux sections suivantes, portant sur la capacité qu'ont les femmes de prendre part à la science et à faire preuve d'autorité, observent une progression similaire ${ }^{19}$.

17. Quoique le cadre de la présente étude ne me permette pas de le faire, il s'avère extrêmement intéressant de comparer les textes originaux à l'usage qu'en fait Suchon. Si certaines références font l'objet d'une réappropriation assez considérable, d'autres sont d'une grande fidélité : c'est le cas, notamment, d'une importante référence (Science, p. I36) aux Actions courageuses et vertueuses des femmes de Plutarque, qui y établit que la vertu est la même chez les hommes et les femmes $(243 \mathrm{c}-\mathrm{d})$. Soulignons que Gournay fait la même référence dans l'Égalité des hommes et des femmes ( I622), p. I3.

18. Dorlin, 200I, p. 42.

19. Si, en matière d'égalité des sexes au Xvir siècle, la postérité a surtout retenu la célèbre formule "l'esprit n'a pas de sexe » de Poulain de la Barre, il est important de noter que les dispositions que Suchon prête aux femmes autant qu'aux hommes ne sont pas strictement intellectuelles. De fait, la question des capacités intellectuelles des femmes n'est introduite que dans la deuxième partie, portant sur la privation de science - ce qui ne revient pas à dire que 
Il faut encore rajouter que la distinction entre la possession d'une capacité (par exemple, la capacité à être libre) et son exercice est essentielle au projet de Suchon. En effet, pour établir qu'il y a privation de quelque chose, il faut pouvoir démontrer la capacité du sujet à posséder ce dont il est question:

Et comme la privation suppose toûjours dans le sujet qui la souffre une capacité naturelle pour acquerir \& pour posseder le bien dont il est privé; je montre par bonnes \& solides raisons, par autoritez, \& par exemples que les femmes sont capables de Liberté, de Science, \& d'Autoritée ${ }^{20}$.

En clair, si les femmes ne possédaient pas de capacité à être libres, savantes et autoritaires, l'absence de ces états ne pourrait être qualifié de privation. Si les femmes n'ont ni liberté, ni science, ni autorité effectives, ce n'est pas parce qu'elles en sont incapables, mais bien parce qu'elles en sont privées.

\section{De l'intérêt bien compris des hommes}

Suchon ne se borne cependant pas à relever un décalage entre la possession de capacités et leur exercice. Elle en diagnostique également les causes et montre que ce décalage tient non seulement à la force de la coutume, mais aussi, et surtout, en un certain intérêt bien compris qui la sous-tend. Les capacités que les femmes possèdent tout autant que les hommes montrent que leur infériorité apparente est tout sauf naturelle: elle tient plutôt à la coutume. Sur ce point, Suchon suit Poulain de la Barre, dont elle se réclame d'ailleurs explicitement:

Ce n'est pas que dans le sentiment d'un moderne [l'auteur de l'égalité des Sexes], c'est une espèce d'injustice de rejeter sur la nature, ce qui n'appartient qu'à la coûtume, parce qu'étant une fois établie l'on croit toûjours qu'elle est bien fondée ${ }^{21}$.

Or, s'il est si difficile de changer la coutume, c'est parce que certains en tirent profit: les hommes, qui, par le pouvoir qui leur incombe, ont bâti une société, des institutions et des lois à leur avantage. Ce triste constat, dont on a à juste titre souligné la radicalitéér, joue un grand rôle dans l'œuvre de Suchon. Comme on le verra dans la section suivante, c'est pour cette raison que son projet doit être mené à bien par les femmes elles-mêmes; l'amélio-

Suchon y attache moins d'importance, mais plutôt qu'elle estime que la question de la liberté doit être traitée en premier lieu, peut-être parce que c'est là où la privation des femmes est la plus flagrante et la plus cruelle (voir Liberté, avant-propos).

20. Suchon, Traité de la morale et de la politique, préface générale. Voir aussi, toujours dans la préface: «[L]'on ne saurait prouver l'absence d'un bien dans un sujet, que l'on ne fasse voir en même temps qu'il est capable d'avoir la possession et la jouissance de ce même bien.»

21. Suchon, Autorité, 67.

22. Cf. Dorlin, 200I ; Desnain, 2006; 20I3 ; Le Doeuff, I998; et Ronzeaud, I975. 
ration de leur condition nécessiterait pour les hommes de renoncer à trop d'avantages. Il est ainsi très clair, pour Suchon, que toute négociation avec les hommes est perdue d'avance:

[L]es femmes perdront toûjours leur cause, parce qu'elles n'ont point d'autres Juges que leurs Maîtres, qui ne veulent jamais changer les coûtumes qu'ils ont introduites. Et bien qu'ils ayant souvent des differens entre eux, ils conviennent tous en ce poinct de rechercher l'abaissement des Personnes du Sexe, \& d'empêcher autant qu'ils peuvent leur élevation ${ }^{23}$.

De fait, si le profit que les hommes retirent de l'asservissement des femmes demeurait encore indéterminé dans la section du Traité de la morale et de la politique consacrée à la liberté et à la contrainte ${ }^{24}$, l'idée se précise dans les sections suivantes. Dans la deuxième partie consacrée à la science, Suchon évoque ainsi l'éducation défaillante des filles à qui on donne accès, au mieux, à des livres pour enfants et à de rares enseignements sur la vie dévote $^{25}$. Même celles qui disposent d'un certain revenu se voient trop souvent refuser la possibilité de l'employer pour acquérir une éducation: on les pousse plutôt à en faire usage de façon superficielle. L'ignorance, bien loin d'être propre aux femmes, est commune à toute la nature humaine; seulement, les hommes sont parvenus à s'en libérer partiellement par l'étude — ce à quoi les femmes n'ont pas accès ${ }^{26}$. Si un doute pouvait persister quant aux causes de cet état de choses - Suchon évoquant parfois simplement la force de la coutume ${ }^{27}$ - elle devient beaucoup plus explicite vers la fin de cette seconde partie, attribuant le manque d'éducation des femmes à un geste délibéré de la part des hommes.

Car, pour Suchon, les hommes, ou du moins ceux qui détiennent le pouvoir, savent fort bien que l'ignorance des femmes ne tient pas à un manque de capacité naturelle; ils ont plutôt entrepris «de les priver de tous les moyens, dont elles pourroient se servir pour se rendre sçavantes ${ }^{28} »$. Son constat est aussi amer qu'impitoyable:

23. Suchon, Science, p. 267.

24. En exposant la nature de la contrainte - une privation de liberté qui acquiert une dimension systématique chez les femmes cloîtrées ou mariées de force - , Suchon évoque certaines motivations condamnables poussant les familles des jeunes femmes à les cloîtrer de force (par exemple, pour se libérer d'une charge financière). Elle ne cible cependant pas spécifiquement les hommes, se bornant à parler des "parents» (voir Suchon, Liberté, p. I28 sq.; voir aussi Liberté, p. I4 I sq. pour un raisonnement similaire sur le mariage, quoique Suchon blâme plus volontiers les jeunes femmes elles-mêmes à ce sujet). L'empire qu'exercent les hommes sur les femmes est cependant évoqué dès le premier traité (Liberté, p. 32 ; pour un propos similaire, voir Autorité, p. II 2).

25. Suchon, Science, p. 24; voir aussi p. 266.

26. Suchon va jusqu'à qualifier la vie des femmes d' "espèce de mort intellectuelle " (Science, p. I65).

27. Voir par exemple Suchon, Science, p. 265.

28. Ibid., p. 264. 
[L]'ignorance du second Sexe n'a point de plus grande cause que l'impérieuse domination du premier, parce qu'ayant établi des Ecôles, des Coléges, des Universitez, des Academies, des Bibliotheques, des Professeurs dans les Sciences, des Regens \& des Maîtres; ils en ont bannis toutes les personnes du Sexe, ne voulant pas qu'elles entrent dans ces palais des Muses [...]; ni qu'elles se rendent Disciples des Sçavans, dont la conférence ne leur est pas libre, si ce n'est pour apprendre en peu de paroles, l'abaissement $\&$ le mépris d'ellesmêmes ${ }^{29}$.

En clair: les hommes, qui détiennent sans aucun doute l'essentiel du pouvoir dans la France du XVII ${ }^{\mathrm{e}}$ siècle de Suchon, entretiennent l'état d'ignorance dans lequel se trouvent les femmes en leur refusant les moyens de s'instruire. Il s'agit moins d'accuser «tous les hommes» de prendre part à un complot contre les femmes que de dénoncer leur responsabilité collective dans l'état d'ignorance de ces dernières, étant donné le mauvais usage qu'ils font des pouvoirs qui leur incombent.

Or la motivation des hommes demeure incertaine si on s'arrête à la deuxième section du traité: pourquoi préférer des compagnes peu ou pas éduquées ? Il serait peut-être plus réaliste d'attribuer l'ignorance des femmes à des coutumes pernicieuses, transcendant les genres et auxquelles les hommes ne prendraient part que très indirectement. Mais Suchon précise sa pensée dans la troisième section, portant sur l'autorité; l'auteure y établit en effet la capacité naturelle des femmes à faire preuve d'autorité et dénonce vivement le mauvais usage que font les hommes de leur autorité sur les femmes - tant dans la sphère publique (au moyen de lois et d'institutions) que dans la sphère privée (étant donné la dépendance forcée des filles envers leur père et des épouses envers leur mari, ces dernières n'étant pas autre chose que "sujettes dans leur propre maison ${ }^{30}$ »). Cette autorité de l'homme sur la femme, que Suchon ne conteste pas comme telle, vient donner lieu à nombre d'abus; la soif de pouvoir des hommes et leur désir de commander (c'est-à-dire, « une passion violente qui se sert souvent de la tyrannie \& de l'injustice pour arriver à ses fins, quand on lui fait resistance ${ }^{31} »$ ), joints à leur position privilégiée, constituent pour elle la source des maux infligés aux femmes. La privation d'autorité imposée aux femmes constitue en fait, pour Suchon, la «cause et principe» des autres privations:

[L]es hommes ne privent les personnes du beau Sexe, des deux premiers avantages [la liberté et la science], que pour les empêcher de prétendre au troisiéme [l'autorité]. Cette maniére franche \& libre qu'ils retiennent pour eux, \& l'empire des Lettres qu'ils s'approprient entiérement, n'étant que pour dominer plus facilement à l'exclusion des femmes, que l'on veut toûjours dans la dependance, parce que l'on prétend que cét état leur est tellement naturel, qu'en tous

29. Suchon, Science, p. 264-265.

30. Suchon, Autorité, avant-propos.

31. Suchon, Autorité, p. 89. 
les siecles elles n'ont jamais fait d'autres figures que celle de soumises \& d'obeissantes ${ }^{32}$.

Ainsi, les hommes retirent un intérêt réel de la contrainte imposée aux femmes et de leur ignorance, puisqu'elles leur permettent de les maintenir plus sûrement sous leur gouverne. Le pouvoir politique, apanage quasi exclusif des hommes, se révèle un instrument de choix pour renforcer cette dépendance; les lois et le droit constituent, pour Suchon, autant de manières d'assurer l'empire des hommes sur les femmes:

Elles sont sujettes à toutes les Polices que les hommes s'avisent d'introduire; aussi la Politique ne les reçoit dans son sein, que comme des sujets passibles où elle exerce ses Loix, ses Ordonnances, ses formalitez, sa justice, son adresse \& tous ses ressorts. Car elles sont tellement dependantes qu'elles n'ont aucune participation à toutes les diverses Autoritez qui se voyent dans le monde ${ }^{33}$.

De fait, si Suchon n'espère pas pouvoir réformer la situation légale des femmes, elle la juge néanmoins fort problématique, les lois contribuant directement à la domination masculine et à l'abaissement des femmes.

L'impossibilité pour les femmes d'exercer leurs capacités naturelles en matière de liberté, de science et d'autorité semble donc tenir, par-delà la force de la coutume, à l'intérêt bien compris des hommes, qui veulent conserver le pouvoir qu'ils détiennent. Ce qui ne revient pas à dire qu'ils n'aiment pas les femmes, mais plutôt qu'ils les aiment mal:

[S]i l'on pénétre un peu dans le secret de cet amour, l'on connoîtra bien-tôt qu'il est renfermé dans leurs propres interêts, \& qu'il ne s'attache qu'à la necessité qu'ils en ont, $\&$ au plaisir $\&$ à l'utilité qu'ils en reçoivent ${ }^{34}$.

De fait, l'amour est, nous dit Suchon, une passion naturelle aussi bien qu'un art dans lequel chacun se croit savant ${ }^{35}$; mais il est difficile d'en faire bon usage. Pour Suchon, l'amour que les hommes portent aux femmes, s'il est bien réel, n'en demeure pas moins (trop souvent) intéressé; la crainte secrète de perdre leur ascendant rend difficile pour eux de veiller à ce que les femmes soient en mesure d'exercer leur liberté et de se consacrer à l'étude de la science. Le véritable amour doit plutôt sortir de lui-même "pour se transporter à son objet » en veillant à l'accomplissement de son bonheur ${ }^{36}$; c'est pourquoi il est si rare et difficile ${ }^{37}$.

32. Suchon, Autorité, p. I I-I2.

33. Suchon, Autorité, p. 88 ; voir aussi p. I07, I32, et Liberté, p. I44, I48.

34. Suchon, Autorité, p. II I.

35. Suchon, Liberté, p. I47.

36. Ibid., p. I I 2.

37. On s'explique mal, à la lecture de passages d'une telle dureté, le commentaire de Hoffman sur la «maladresse, [la] naïveté, [la] timidité » de Suchon (Hoffman, I978, p. 276). 


\section{L'élévation des femmes, un projet politique}

Il convient maintenant de passer à la dimension proprement politique du projet de Suchon, c'est-à-dire l'élévation des femmes. On verra que cette entreprise ne peut être accomplie que par les femmes elles-mêmes, sans le concours des hommes. Le récit de la chute confirme, pour Suchon, l'inévitabilité pour la femme de se trouver en position de soumission par rapport à l'homme, qui, comme on sait, fait bien mauvais usage de son autorité. Le pouvoir détenu par les hommes en vient même à les corrompre, les rendant impropres à prendre part au perfectionnement des femmes.

\section{Les fermmes comme premières destinataires de Suchon}

On comprend déjà pourquoi Suchon, à la différence de la plupart des auteurs de la querelle, s'adresse d'abord et avant tout aux femmes, son projet ne pouvant être mené à bien que par elles. Ainsi, contrairement à Poulain de la Barre, Suchon n'espère pas changer les mentalités: si le premier veut montrer que l'inégalité des deux sexes n'est pas autre chose qu'un préjugé dont il faut se débarrasser pour parvenir à des connaissances claires et distinctes ${ }^{38}$, la seconde ne semble pas convaincue que la recherche de connaissances vraies puisse constituer une motivation suffisante pour amener les hommes à renoncer à un préjugé qui sert si bien leurs intérêts. De fait, quoique Poulain et Suchon s'entendent sur le rôle prépondérant joué par la coutume dans la perpétration d'idées préconçues sur les femmes, Suchon explique ultimement cette force de la coutume par l'intérêt qu'y prennent ceux qui détiennent le pouvoir. Poulain, quant à lui, évoque bien cette cause ( $\mathrm{Si}$ on cherche sur quoi sont fondées toutes ces opinions diverses, on trouvera qu'elles ne le sont que sur l'intérêt, ou sur la coutume; et qu'il est incomparablement plus difficile de tirer les hommes des sentiments où ils ne sont que par préjugés... $\left.{ }^{39} »\right)$, mais son analyse insiste davantage sur la force de la coutume et ses effets sur les mentalités - ainsi que, dans la deuxième partie de L'égalité des deux sexes, sur les preuves erronées des savants. De fait, pour Poulain, un examen attentif de la croyance en l'infériorité des femmes devrait logiquement résulter en l'élimination de cette croyance:

Cependant cette persuasion comme la plupart de celles que nous avons sur les coutumes et sur les usages n'est qu'un pur préjugé, que nous formons sur l'apparence des choses, faute de les examiner de près, et dont nous nous détromperions, si nous pouvions nous donner la peine de remonter jusqu'à la source $[\ldots]^{40}$.

38. Poulain, 20I I, p. 53.

39. Poulain, 20I I, p. 60; je souligne en italique. Un passage similaire de la préface de L'Égalité des deux sexes omet purement et simplement la référence à l'intérêt que certains peuvent prendre à entretenir un préjugé: "[Le vulgaire] n'ayant pour fondement de ce qu'il croit, que la Coutume et de légères apparences, [...]» (ibid, p. 54 ; je souligne en italique).

40. Poulain, 20I I, p. 63. 
Toute l'entreprise de Poulain vise ainsi, d'une part, à réfuter ce préjugé tenace (en s'adressant, pour ce faire, tant à l'homme du commun qu'au savant) - et d'autre part, à en combattre les effets en encourageant les femmes à se prévaloir de l'éducation à laquelle elles devraient avoir droit ${ }^{41}$. Le projet de Poulain mise donc essentiellement sur l'éducation, à tout point de vue.

Si Suchon reconnaît également l'importance capitale pour les femmes de bénéficier d'une instruction faisant honneur à leurs capacités intellectuelles, l'éducation ne constitue pourtant qu'une dimension de son projet. Suivant l'articulation du Traité de la morale et de la politique, la science est placée sur un pied d'égalité avec la liberté et l'autorité; et de fait, les buts de Suchon sont non seulement éducatifs, mais aussi, et surtout, politiques ${ }^{42}$. Pour elle, il appartient aux femmes, individuellement et collectivement, de se réapproprier non seulement la science, mais aussi la liberté et l'autorité dont on les prive. C'est pour cette raison qu'elle s'adresse d'abord et avant tout aux femmes, comme en témoigne la préface générale du Traité:

Je n'ay point eû d'autre intention en tout ce traité que d'inspirer aux personnes $d u$ Sexe des sentimens genéreux \& magnanimes afin qu'elles se puissent garentir d'une contrainte servile, d'une stupide ignorance, \& d'une dépendance basse \& ravalée. Ce qu'elles pourront faire tres-facilement si elles suivent ce qui est inseré à propos sur chacun de ces sujets $[\ldots]^{43}$.

On remarque en effet que les arguments démontrant l'égalité de capacité des hommes et des femmes ne constituent, somme toute, qu'une partie du traité de Suchon, qui cherche moins à convaincre les hommes du bienfondé de ses thèses qu'à mobiliser les femmes autour de son projet d'amélioration de la condition féminine. Comme on l'a vu, l'intérêt que prennent les hommes à l'asservissement des femmes est trop grand pour que les premiers puissent contribuer de façon significative à l'élévation des secondes. C'est donc à elles de mener ce projet à bien, afin d'éviter de le faire dépendre du bon vouloir de ceux que Suchon appelle volontiers leurs «maîtres».

41. Alors que le premier volet du projet de Poulain fait l'objet de son traité le plus célèbre, De l'égalité des deux sexes, le second est surtout développé dans L'éducation des dames. À ce sujet, on consultera avec profit l'introduction de Marie-Frédérique Pellegrin à son édition des trois traités de Poulain de la Barre (20 I I, p. I I sq.).

42. Suchon est bien sûr loin d'être la seule participante de la querelle à espérer changer le statut social des femmes. Cependant, elle semble être seule à préconiser une solution qui ne soit pas d'abord et avant tout éducative et qui soit en outre menée à bien par les femmes ellesmêmes. Fauré expose bien cette particularité: «Dans le traité de Gabrielle Suchon, la raison faisait partie des capacités intellectuelles féminines, mais n'était point investie d'une valeur et d'une efficacité singulières à l'endroit de l'émancipation des femmes." Fauré, I985, p. I30.

43. Suchon, Traité de la morale et de la politique, préface générale; je souligne en italique. 


\section{Une différence sexuelle héritée d'Adam et Ève}

Il semble donc que le projet de Suchon repose sur une certaine conception de la différence sexuelle qu'il vaut la peine de tirer au clair. Il va sans dire que cette question mériterait de faire l'objet d'une étude à part entière; je me bornerai à en souligner les aspects qui affectent directement le projet politique de Suchon.

On a vu que, pour Suchon, la différence sexuelle ne peut être située au niveau des capacités fondamentales de l'être humain en matière de liberté, de science et d'autoritée ${ }^{44}$. Suchon reconnaît bien l'existence de différences physiologiques entre les deux sexes, qui leur confèrent des tempéraments distincts mais ne sont pas significatives sur le plan intellectuel ou moral. On peut penser, par exemple, à une certaine propension à la mélancolie qu'elle prête aux femmes, leur tempérament ayant «beaucoup moins de chaleur \& plus d'humidité que celui des hommes; ce qui les rend plus disposées à la melancholie $^{45}{ }$. Or la mélancolie n'est pas en elle-même une mauvaise chose (Suchon y voit même une potentielle source de sainteté); l'important est qu'elle soit bien réglée et ce, chez les hommes comme chez les femmes. En refusant d'accorder un rôle significatif à la différence entre les tempéraments, Suchon se démarque ainsi des détracteurs du sexe féminin, mais également d'auteures comme Lucrezia Marinella qui utilisent cette différence pour défendre la supériorité des femmes ${ }^{46}$. Elle revient par ailleurs sur cette différence entre les tempéraments dans le Petit traité de la faiblesse, de la légèreté et de l'inconstance qu'on attribue aux femmes mal à propos annexé au Traité de la morale et de la politique, niant qu'elle ait une quelconque répercussion sur le plan de la force physique et utilisant pour ce faire l'exemple de l'accouchement:

Il semble que la nature même s'oppose aux outrages que l'on fait aux femmes de les faire passer pour foibles; puisqu'elle les a destinées à la souffrance des plus insupportables douleurs qui sont celles de l'enfantement, \& aux fâcheuses peines qu'il faut endurer en la nourriture $\&$ éducation de leurs enfans ${ }^{47}$.

44. Ronzeaud qualifie l'œuvre de Suchon de « décevante » dans la mesure où elle tente d'abolir la différence sexuelle sur le plan des capacités susmentionnées; car pour lui, cette différence est évidente («Tentant de prouver la ressemblance du sexe faible et du sexe fort, G. Suchon conforte celui-ci dans sa position de modèle supérieur. Elle essaye d'abolir la différence ontologique à partir de trois arguments: intelligence similaire, égalité corporelle, égalité spirituelle, tous trois voués à l'échec puisque cette différence existe réellement» Ronzeaud, I975, p. 28.) On regrette que Ronzeaud n'ait pas jugé bon de fournir des arguments appuyant sa thèse en la matière.

45. Suchon, Liberté, p. 165.

46. Marinella, La nobiltà et l'eccellenza delle donne: co'diffetti, et mancamenti de gli huomini (I60I). Soulignons que Marinella est la seule auteure de la querelle des femmes à avoir une entrée à son nom dans le Dictionnaire historique et critique de Pierre Bayle (I697). Les noms de Marie de Gournay, Jacquette Guillaume, François Poulain de la Barre et Anna Maria van Schurman sont d'ailleurs évoqués dans cette entrée.

47. Suchon, Petit traité, p. 6. Comparer ici avec Poulain de la Barre qui, s'il ne prête pas à la femme une force moindre que celle de l'homme en général, considère néanmoins que la grossesse compromet les forces de la femme (voir De l'égalité des deux sexes, 20I I, p. 65 et I 28). 
Malgré le peu d'importance des différences physiologiques, Suchon reconnaît une autre forme de différence sexuelle, laquelle est essentielle à la bonne compréhension de son projet: une différence induite par Dieu et qui a de sérieuses répercussions sur les rapports entre les sexes. À cet égard, Suchon se distingue explicitement de Poulain:

Quoy qu'un Auteur de ce tems [L'auteur de l'égalité des Sexes] prétende modérer la malédiction que Dieu fulmina contre Eve; [...] \& que pour favoriser son parti, il s'aide d'une version Hebraïque qui semble un peu en adoucir la rigueur; neanmoins la plus grande partie des Sçavans qui suivent celle de saint Jerôme, nous marquent expressément \& sans glose cette soûmission \& dependance $[\ldots]^{48}$.

De fait, ce n'est pas un hasard si cette question est soulevée dans le cadre de la section du Traité consacrée à l'autorité. Suchon s'y attelle en effet à démontrer que, malgré cette soumission fondamentale, la femme a tout de même une capacité à faire usage d'autorité et qu'elle devrait être en mesure de l'exercer. Le rapport hiérarchique entre les sexes est maintenu, en ce sens où Ève doit une certaine soumission à Adam; mais Ève doit demeurer une compagne et non une servante, car tous deux partagent une capacité fondamentale en matière d'autorité - de fait, Dieu leur a conféré à tous deux un pouvoir égal sur les autres animaux ${ }^{49}$. C'est pourquoi l'autorité unilatérale du mari sur sa femme est problématique; dans ce cas, l'autorité est utilisée à mauvais escient. Suchon se réclame à cet égard d'Aristote:

Si les hommes suivoient le conseil d'Aristote, quand il veut que le gouvernement des familles soit Aristocratique; c'est à dire celui des sages $\&$ des gens de bien, où chacun s'employe selon sa capacité; les femmes vivroient plus heureuses $\&$ avec moins de chagrin, mais comme ils veulent toûjours avoir l'autorité entiere sans y souffrir la moindre division; de là vient que c'est une usurpation plûtôt qu'un éfet de leurs privileges, qui ne les dispensent pas de suivre les principes de la raison ${ }^{50}$.

Suchon en retire que les maris usent bien mal de leur autorité, se l'accaparant tout entière alors qu'ils devraient plutôt consulter leurs femmes et même en faire "leurs égales dans la conduite de la maison". Notons que Suchon infléchit quelque peu le propos d'Aristote, qui est loin de suggérer que l'autorité familiale doive être partagée entre le mari et sa femme suivant leurs aptitudes respectives ${ }^{51}$. Pour celui que Suchon appelle volontiers le

48. Suchon, Autorité, p. 9I. Il est intéressant de noter que Suchon réfère ici non à un passage de Égalité des deux sexes, mais bien de Excellence des hommes contre l'égalité des sexes (Poulain, 20II, p. 304 sq.).

49. Suchon, Autorité, p. 7.

50. Suchon, Autorité, p. 92-93. Suchon réfère ici au passage en I I60b32 - I I6IaI de l'Éthique à Nicomaque (qu'elle connaissait sous le nom de Morale).

51. Étant donné le nombre et la précision générale des références de Suchon à Aristote, il est peu probable qu'il s'agisse là d'une erreur de lecture (qui serait assez considérable); il 
"prince des philosophes», la communauté formée par le mari et sa femme doit être comprise par analogie avec le régime aristocratique, mais parce que l'autorité revient naturellement au mari par son mérite, comme en témoigne le passage de l'Éthique à Nicomaque auquel Suchon réfère:

Le gouvernement du Mary, \& de la Femme tient plus de celuy des gens de bien, que nous avons appellé Aristocratique, chacun s'employant à proportion de sa capacité [kat'axian] ${ }^{52}$, le Mary presidant à la principale administration, comme le plus capable, \& la Femme au reste des affaires qui n'excedent pas la portée de sa conduite ${ }^{53}$.

Il est cependant exact qu'Aristote préconise de céder une partie de la gestion du foyer à la femme, comme l'indique la dernière partie de la citation reproduite ici. S'il ne s'agit pas tout à fait d'un partage d'autorité au sens où l'entend Suchon, la suite du passage de l'Éthique à Nicomaque vient appuyer sa condamnation des abus d'autorité: Aristote y souligne que si le mari décide de tout en souverain, il transforme alors son ménage en oligarchie, car «c'est par usurpation, \& non pas par merite que le Mary prend l'authorité toute entiere ${ }^{54} »$. On peut en retirer que Suchon, tout en acceptant l'idée d'une certaine soumission de la femme à son mari, considère qu'une gestion juste et efficace du foyer doit prendre en considération l'égale capacité naturelle des époux à commander et résulter en un partage des responsabilités conséquent. La soumission recommandée aux femmes envers leur mari ne leur serait d'ailleurs "pas tant onéreuse \& penible, si les hommes se servoient de leur autorité selon la volonté de Dieu, \& comme il leur est commandé dans les saintes Lettres $[\ldots]^{55}{ }$. Cette différence entre les sexes héritée d'Adam et Ève s'avère ainsi très importante pour Suchon. Sans être naturelle en un sens biologique, cette différence n'en est pas moins induite par Dieu et réitérée par l'Église; en ce sens, il n'est pas possible pour

semble plus vraisemblable que Suchon se soit délibérément approprié le propos d'Aristote en adaptant le texte original à ses fins. Ce type d'appropriation, qui amuse Ronzeaud (I975, 29), n'est pourtant pas inhabituel dans la querelle ni, de façon plus large, dans l'histoire de la philosophie: on n'a qu'à penser à la célèbre récupération des idées platoniciennes effectuée par Kant, qui prétendait par là avoir compris Platon "mieux que lui-même » (Critique de la raison pure, $\left.\mathrm{A}_{3} \mathrm{I}_{4} / \mathrm{B}_{370}\right)$.

52. La récente traduction de R. Bodéüs rend plutôt kat'axian par «mérite». Je remercie Marguerite Deslauriers pour ces indications.

53. Aristote, Éthique à Nicomaque, II6ob32-34. Nous citons ici la traduction de C. Catel ( 1644 , p. 369): le choix des termes de Suchon (par exemple, dans l'extrait cité: "gens de bien ", "capacité ») suggère qu'il s'agit bien de l'édition à laquelle elle réfère. Vu ce qu'on sait de sa formation, il est peu probable qu'elle ait été en mesure de lire le grec ancien ou le latin, et plus plausible qu'elle ait eu recours à une traduction française de l'Éthique à Nicomaque. Les autres traductions françaises disponibles à la fin du XVII ${ }^{\mathrm{e}}$ siècle étaient celles de Nicole Oresme (I488), beaucoup plus fragmentaire, et celle de Philippe Le Plessis (I 553), qui n’inclut pas le passage cité.

54. Aristote, Éthique à Nicomaque, I I6ob36 - I I6ra I.

55. Suchon, Autorité, p. 92. 
Suchon de penser l'homme et la femme comme interchangeables, malgré leur égale capacité à exercer l'autorité ${ }^{56}$. On verra dans la section suivante que cette différence fondamentale vient renforcer la nécessité, pour les femmes désirant faire un plein usage de leur autorité naturelle, de se soustraire à toute relation avec les hommes et d'endosser plutôt le modèle du célibat volontaire prôné par Suchon.

\section{La corruption des hommes et ses conséquences pour les femmes}

On a vu que malgré des capacités naturelles égales en ce qui a trait à la liberté, à la science et à l'autorité, les femmes se trouvent désavantagées par rapport aux hommes et que la faute en incombe à ces derniers, qui trouvent leur compte dans cette subordination. Il apparaît ainsi que les hommes, malgré les avantages apparents dont ils bénéficient en ce qui a trait à l'exercice de leurs capacités naturelles, sont gravement corrompus. La raison en tient, semble-t-il, au rapport hiérarchique entre les sexes hérité du récit de la chute évoqué plus haut. En effet, comme les hommes sont placés d'emblée en position de pouvoir et que leur liberté extérieure est rarement restreinte à la manière de celle des femmes, leurs occasions de mal faire sont plus nombreuses et plus préjudiciables:

Personne ne sçauroit jamais nier [...], que les hommes n'ayent incomparablement plus de pouvoir \& plus d'occasions pour tomber dans le peché que les femmes, $\&$ que les plus grands desordres qui se voyent tous les jours ne soient leurs ouvrages $\&$ les effets de leurs plus ordinaires pratiques ${ }^{57}$.

En clair: les hommes ne sont ni plus ni moins accablés par les passions que les femmes, mais ont une plus grande facilité qu'elles à leur donner libre cours. On peut ainsi dire que le décalage entre la possession d'une capacité et son exercice est également présent chez les hommes; seulement, alors que les femmes ont du mal à exercer leurs capacités, les hommes font un mauvais usage des leurs. Ce mauvais usage est particulièrement frappant en ce qui a trait à l'autorité qu'ils détiennent sur les femmes: les hommes "abusent assez souvent de leur pouvoir, lors qu'ils commandent par caprice \& sans considération» - sacrifiant les femmes à leur ambition, leur avarice, leur jalousie, voire même à leur débauche ${ }^{58}$. Cette domination unilatérale des

56. Les propos de Suchon elle-même, comme sa dissociation explicite d'avec la position plus égalitaire de Poulain à cet égard, me laissent croire qu'elle regarde l'asymétrie du rapport d'autorité entre hommes et femmes comme un donné aussi inévitable qu'incontestable. On peut cependant se demander si, sans aller jusqu'à contester ouvertement cette asymétrie, elle la juge réellement souhaitable - puisque ne tenant pas compte de l'égale capacité naturelle de l'homme et de la femme à commander. Y répondre par la négative aurait évidemment des conséquences très lourdes sur le plan religieux. Cette question, qui dépasse le cadre de la présente étude, sera, on l'espère, approfondie ailleurs.

57. Suchon, Science, p. I76.

58. Suchon, Autorité, p. 92. 
femmes se répercute jusque dans les lois et le droit, dont les effets néfastes ont été évoqués plus haut. Or, du fait de cette soif de pouvoir conduisant à un mauvais exercice de ses capacités, l'homme nuit à la femme tout en trahissant sa propre nature - ce pourquoi il ne peut constituer un modèle à suivre pour les femmes:

les hommes ont tellement dégéneré de l'estime $\&$ de la tendresse qu'ils doivent aux personnes du Sexe, que l'on peut dire avec verité, qu'ils ont renoncé à une partie d'eux mémes, par les mépris qu'ils font continuellement des femmes ${ }^{59}$.

Malgré ce portrait pessimiste de la gent masculine, Suchon ne suggère pas que tous les hommes soient à ce point dégénérés: elle affirme, dans sa préface, ne pas vouloir causer de tort à l'estime «que l'on doit à tous ceux qui sont véritablement des hommes [; ] [c'est-à-dire] des sages, des judicieux, des sçavans, des habiles, des debonnaires, $\&$ des prudens ${ }^{60} »$. Elle recourt par ailleurs volontiers à des autorités et à des exemples masculins. Seulement, tout comme les femmes exemplaires dont certains auteurs de la querelle font l'éloge sont, d'une certaine façon, des héroïnes qui ont su surmonter les difficultés de leur condition, on peut deviner que les hommes "véritables" sont plus rares qu'il n'y paraît. Quant à savoir si les hommes corrompus sont récupérables, il s'agit d'une question qu'elle laisse volontairement en suspens, puisque son traité se préoccupe de l'élévation des femmes et pas de celle des hommes - qui nécessiterait un tout autre type de travail étant donné le mal différent dont ils sont atteints. Quoiqu'il en soit, au vu de l'ampleur de la corruption à l'œuvre, on peut inférer que la majorité des rapports entre hommes et femmes seront néfastes à ces dernières.

En raison de l'intérêt indéniable que prennent les hommes (ou du moins la plupart d'entre eux) à la subordination des femmes et de l'autorité qu'ils détiennent sur elles, on ne peut que se tourner vers ces dernières pour espérer une amélioration de leur condition. Dans l'impossibilité où elles se trouvent de changer les institutions et les lois régissant ces rapports — soulignons que la démocratie ne pouvait représenter une solution prometteuse pour une femme du XVII ${ }^{e}$ siècle ${ }^{61}$ - la solution de Suchon consiste plutôt à les isoler de tout rapport avec les hommes pour permettre leur élévation. Comme on le verra, il s'agit d'une solution drastique mais nécessaire, en ce qu'elle vient soustraire les femmes à la soumission, légitime ou non, qu'elles doivent à ceux du "premier sexe » — en leur permettant par le fait même de s'instruire et de développer leurs capacités à la liberté et à l'autorité ${ }^{62}$.

59. Suchon, Petit traité, préface.

60. Suchon, Traité de la morale et de la politique, préface générale.

61. Suchon, Autorité, p. 24.

62. Le fait de chercher à soustraire les femmes à toute forme de relation avec les hommes peut paraître radical, voire même exagéré. Pourtant, pour une femme du XvII siècle qui, bien qu'issue d'une bonne famille, ne disposait pas des avantages et des ressources des femmes nobles, il y avait au final peu de façons d'échapper aussi efficacement à un problème de nature 


\section{L'élévation des femmes}

Il est à présent possible, sur les bases du projet de Suchon, d'en examiner la concrétisation: c'est-à-dire, la condition de neutraliste devant permettre aux femmes de se perfectionner en les soustrayant à toute forme de rapport de pouvoir avec les hommes.

\section{La condition de neutraliste}

Pour rendre possible l'élévation des femmes, il semble que la seule solution consiste à les encourager à adopter un mode de vie indépendant, en réduisant autant que possible leurs contacts avec les hommes. Cette implication du Traité de la morale et de la politique fait l'objet du second traité de Suchon, Du célibat volontaire, qui ménage un nouveau statut pour les femmes: celui de "neutraliste». Je montrerai ici que c'est cet état, et celui-là seul, qui permet une réelle élévation des femmes en leur procurant le détachement nécessaire à une vie aussi pieuse qu'accomplie, tout en leur permettant d'être réellement utiles à la société par l'exercice plein et entier de leurs capacités naturelles. Avec la condition de neutraliste, l'égalité des sexes se trouve pour ainsi dire transcendée: non seulement les femmes ne sont plus inférieures, mais elles sont même en mesure de se rendre supérieures aux hommes.

La condition de neutraliste créée par Suchon repose, comme le nom l'indique, sur un état de neutralité, symbolisant le fait de ne pas endosser un état plutôt qu'un autre; il s'agit en effet d'une vie sans engagement, ou célibat volontaire $^{63}$. De fait, Suchon cherche à poser ce célibat volontaire comme une solution de rechange au cloître et au mariage, les deux états les plus courants pour une femme de son temps. Le célibat volontaire «renferme tous les autres états en puissance, sans les mettre en pratique ${ }^{64}$ », puisqu'il est sans engagement - Suchon entendant par «engagement » l'obligation de demeurer toujours dans une même condition. Il s'agit là d'une différence majeure avec l'état de religieuse, qui comporte précisément une telle obligation. Le célibat volontaire vise ainsi à soustraire les femmes aux contraintes que représentent le mariage et le cloître, et à leur permettre un plein exercice de leur liberté. Suchon s'inspire davantage de l'état de veuve, lequel permettait peut-être le plus de liberté aux femmes de son temps; elle considère même que les neutralistes sont "celles qui sont veritablement veuves, c'est à

systémique: celui des rapports de pouvoir entre hommes et femmes — un problème qui, comme on le sait, devait avoir une postérité certaine dans le domaine des études féministes.

63. Dunn-Lardeau souligne que Moderata Fonte et Marie de Gournay faisaient déjà l'apologie, avant Suchon, d'une forme moins élaborée de célibat volontaire (Dunn-Lardeau, 2002). Desnain souligne, quant à elle, l'influence probable de Nicolas Cassin sur la conception de Suchon — avec, bien sûr, cette différence que Cassin s'adresse aux hommes et non aux femmes (Desnain, 2009).

64. Suchon, Le célibat volontaire, p. 2. 
dire parfaitement libres de toutes les affections étrangeres, \& entierement indifferentes pour tous les engagemens du monde ${ }^{65}{ }$. De fait, alors que la fin du mariage est la procréation et que celle de l'état séculier est de louer Dieu, la fin du célibat volontaire est de vivre sans engagement, de manière "abstraite $\&$ separée des maximes du monde ${ }^{66} »$. Ce détachement est, comme on le verra à présent, ce qui rend la condition de neutraliste supérieure à toutes les autres et permet l'élévation des femmes qui l'adoptent.

\section{Un plein usage des capacités naturelles}

L'élévation des femmes implique, pour Suchon, trois aspects: (i) servir Dieu; (ii) se perfectionner elles-mêmes; et (iii) être utiles à autrui ${ }^{67}$. Encore une fois, on voit que le perfectionnement des femmes ne profite pas qu'à elles seules; toute la société doit en bénéficier. Il est par ailleurs clair que c'est par un usage adéquat (et donc non restreint) de leurs capacités naturelles que les femmes pourront satisfaire à ces conditions. Or, comme on le verra à présent, la condition de neutraliste s'avère être la seule permettant aux femmes d'exercer pleinement leur liberté et leur autorité, mais aussi de se consacrer à la science - et, conséquemment, de faire un usage optimal de leurs capacités naturelles.

En ce qui concerne la liberté, il est manifeste que la condition de neutraliste, compte tenu de l'absence d'engagements qui la caractérise, doit permettre une liberté extérieure sans pareille aux femmes qui l'adoptent. Elle remplit cependant une fonction tout aussi importante en ce qui a trait à la liberté intérieure de l'âme, forme de détachement qui s'exprime notamment par une liberté de cœur, caractérisée dans le Traité de la morale et de la politique comme "un cœur sans amour, sans haine et sans désir ${ }^{68}$ ». De fait, Suchon insiste sur la nécessité pour les neutralistes d'être libres intérieurement aussi bien qu'extérieurement, ce qui laisse penser que sa conception de la "vie sans engagement", qu'elle décrit d'abord comme une liberté de condition (et donc extérieure), implique également une forme de détachement intérieur ${ }^{69}$. Elle admet ainsi au rang des neutralistes celles qui, n’ayant pas les moyens de vivre sans travailler ${ }^{70}$, parviennent malgré tout à mener

65. Suchon, Le célibat volontaire, p. 23. Cette condition était déjà suggérée dans Liberté, p. I49, Suchon y encourageant les femmes libres à demeurer dans cet état.

66. Suchon, Le célibat volontaire, p. 29

67. Ibid., p. 354; voir aussi L'avertissement.

68. Suchon, Liberté, p. 65.

69. Dans sa fort intéressante étude intégrant Suchon à une histoire de la notion d'autonomie en philosophie, Shapiro associe la "vie sans engagement» à une vie libre, mais en mobilisant uniquement l'aspect extérieur de la théorie de la liberté de Suchon (voir Shapiro, à paraître). Les développements suivants me laissent croire qu'il faudrait également y intégrer sa dimension intérieure.

70. Il vaut la peine de souligner que la condition de neutraliste est sans contredit ouverte aux femmes de toutes classes sociales; Suchon insiste souvent sur l'importance d'y inclure celles 
une vie sainte, «qui n'est autre que la liberté du cœur ${ }^{71}$ ». Il s'agit en fait d'une dimension très importante du célibat volontaire, que Suchon vient souligner dans une définition ultérieure:

le Celibat est un amour passionné d'un parfait dégagement, d'une veritable innocence \& d'une solitude abstraite \& separée des choses du monde, dans le dessein de se rendre victorieux des foiblesses $\&$ des passions humaines ${ }^{72}$.

Dans ce contexte, on comprend d'autant mieux pourquoi il est nécessaire pour les femmes de se soustraire à la compagnie des hommes afin de se perfectionner: Suchon considère nécessaire de limiter autant que possible les attachements terrestres afin de ne rien aimer avant $\operatorname{Dieu}^{73}$. Une vie retirée, à l'abri des passions immodérées, semble donc nécessaire pour satisfaire cet aspect de la condition de neutraliste - tant en regard des passions tristes causées par les méfaits des hommes que de celles plus joyeuses liées à l'amour. Il ne s'agit pas de vivre en ermite pour autant; Suchon se montre favorable à la vie en communauté et souligne la valeur des amitiés sincères et véritables au chapitre XXII du Célibat volontaire ${ }^{74}$.

Les formidables possibilités qu'offre la vie sans engagement pour l'étude sont, quant à elles, assez évidentes: les neutralistes se trouvant dégagées de leurs habituelles fonctions en société de mères, de religieuses, etc., elles ne peuvent qu'avoir davantage de temps à consacrer à leur instruction. La capacité des femmes à se consacrer à la science ayant été établie dans le Traité de la morale et de la politique, Suchon élabore dans Le célibat volontaire un ambitieux programme d'études pour les neutralistes, comprenant morale, physique, métaphysique, grammaire, rhétorique $^{75}$, arithmétique et $\operatorname{arts}^{76}$. La question de savoir si cette instruction doit demeurer une entreprise solitaire ou au contraire prendre une forme collective mérite d'être soulevée: Suchon se rend bien compte que les femmes ne seront pas de sitôt admises

qui ont à travailler pour gagner leur vie et distingue volontiers les éventuelles communautés de neutralistes des communautés religieuses à cet égard, ces dernières étant trop souvent réservées aux femmes nobles ou riches (Suchon, Le célibat volontaire, p. I7).

71. Suchon, Le célibat volontaire, p. I 5.

72. Ibid., p. 65 I.

73. Voir notamment Suchon, Science, p. 7I.

74. Suchon, Le célibat volontaire, p. 399 sq.; voir aussi Autorité, p. 48. Pour Suchon, l'amitié est supérieure à l'amour fondant le mariage et la famille, car elle n'implique pas la dimension d'obligation et d'engagement permanent qu'ils représentent. De fait, Suchon parle souvent, en des termes assez durs, des nombreuses peines et même des maux physiques qu'encourent celles qui se marient et enfantent. Par exemple, dans Le célibat volontaire: «Les maladies qu'elles souffrent durant neuf mois qu'elles les portent dans leur sein, sont les avant-coureurs de ce qu'elles doivent endurer, en les mettant au jour» (Suchon, Le célibat volontaire, p. 273). La neutraliste, allègue-t-elle, sera plus utile, heureuse et accomplie en s'occupant des enfants qui ne sont pas biologiquement siens (Suchon, Le célibat volontaire, p. 271-272).

75. Desnain suggère que l'insistance de Suchon sur la rhétorique vise à donner aux femmes les moyens de prendre part à des débats publics (Desnain, 20I3, p. 262).

76. Suchon, Le célibat volontaire, p. 352 sq. 
dans les institutions d'enseignement des hommes — ce qu'elle ne juge du reste pas souhaitable, étant donné l'influence néfaste qu'ils pourraient exercer sur elles - et plaide plutôt pour la fondation de collèges, d'universités et d'académies réservées aux femmes ${ }^{77}$. Tant que ces institutions n'auront pas été créées, les femmes devront se résoudre à s'instruire de façon autonome, tout en interagissant les unes avec les autres dans la mesure du possible. Plutôt que de revendiquer ouvertement des institutions d'enseignement pour les femmes, Suchon préconise ainsi à moyen terme la formation de sociétés où les femmes pourraient raisonner, argumenter et se disputer entre elles ${ }^{78}$; on peut en déduire que les neutralistes auraient avantage à se rassembler de la sorte. Leur accomplissement intellectuel ne sera cependant complet que grâce à l'intervention de maîtres:

l'étude des Sciences \& des Arts ne se peut faire sans l'aide des Maîtres, dont la parole comme un fleau porte les coups reiterés de l'instruction dans l'esprit de leurs Disciples: \& avec le fer de leur correction ils retranchent tout ce qui peut empêcher les plantes qu'ils édifient, de porter les fleurs \& les fruits des belles connaissances ${ }^{79}$.

Cette fonction du maître suppose donc un cadre d'enseignement plus formel pour le perfectionnement de la neutraliste, qui semble nécessiter, pour être complété, des institutions appropriées.

Enfin, la capacité des femmes à exercer l'autorité ne peut que bénéficier de leur éloignement d'avec les hommes: comme on l'a vu plus haut, la seule forme de différence sexuelle que Suchon reconnaisse réside dans la soumission que les femmes doivent aux hommes dans le mariage, devant Dieu. Qu'on approuve ou non cette soumission qui remonte à Adam et Ève, elle demeure, pour Suchon, inévitable — tant et aussi longtemps que les femmes entretiendront des relations avec les hommes à travers des formes d'engagement comme le mariage. On a vu que Suchon considère que la plupart de ces relations sont néfastes aux femmes, étant donné les abus quasi inévitables qui résultent du pouvoir plus grand conféré aux hommes en société et menant à leur propre corruption. Or, même en cas de mariage heureux, on peut inférer que la condition de la neutraliste sera préférable à celle de la femme mariée - car seule la première peut exercer pleinement sa capacité à l'autorité, la seconde devant une certaine soumission à son mari. Il ne s'agit pas d'en conclure que toutes les femmes devraient renoncer au mariage et aux autres formes d'engagement: Suchon est bien consciente que la bonne marche de la société requiert une pluralité de vocations. Elle considère néanmoins que celles qui s'abstiennent de tout engagement (ce qui est par ailleurs très difficile) sont aussi celles qui parviendront à se rendre les

77. Suchon, Science, p. 266.

78. Ibid., p. 268.

79. Suchon, Science, p. 258-259; voir aussi Le célibat volontaire, p. 348. 
plus utiles en société, et aux yeux de Dieu, par l'exercice de leur autorité. Les neutralistes en feront usage en prenant part à l'éducation d'autrui, mais aussi en contribuant à l'élaboration de lois plus justes. On peut en retirer que Suchon vise, à long terme, une participation active des femmes en politique $^{80}$; mais à plus court terme, cet exercice de l'autorité pourrait très bien s'effectuer autrement en société, par exemple dans des institutions réservées aux neutralistes ou dirigées par elles. Or cette autorité ne peut être exercée pleinement qu'en se soustrayant à toute forme d'engagement envers les hommes, compte tenu de la soumission qui leur est due ${ }^{81}$.

\section{Conclusion}

Suchon élabore ainsi un projet qui fait écho à plusieurs préoccupations propres au contexte de la querelle des femmes, notamment en ce qui a trait à sa démonstration de l'égalité des capacités des deux sexes. Et comme bon nombre de ses prédécesseurs et contemporains, elle vise ultimement à permettre aux femmes de se perfectionner en faisant bon usage de leurs capacités. Ce projet prend cependant une tournure peu habituelle par sa forme et son caractère exclusivement féminin. Suchon a, on l'a vu, peu d'espoir de pouvoir changer les idées reçues à propos des femmes; elle attribue la force de la coutume à un intérêt masculin bien compris, les hommes retirant certains avantages du fait de maintenir les femmes dans un état de subordination. Au vu de cet intérêt, il lui semble préférable d'amener les femmes à se perfectionner sans compter sur le concours des hommes. Par ailleurs, on a vu que, suivant le récit de la chute endossé par Suchon, les hommes se trouveront toujours en position d'autorité vis-à-vis des femmes. Or les pouvoirs plus grands qu'ils détiennent, joints à cette position privilégiée, les mènent souvent à abuser de leur autorité au détriment des femmes. En raison de ces débordements et, de façon plus large, pour soustraire ces dernières à tout rapport d'autorité vis-à-vis des hommes, Suchon préconise que l'élévation des femmes prenne place dans un contexte non mixte. C'est ce qui motive la création d'une nouvelle condition, celle de neutraliste, devant permettre aux femmes un plein usage de leurs capacités naturelles. Par l'entremise du célibat volontaire, les femmes devraient ainsi parvenir à acquérir une forme de supériorité sur le reste de l'humanité.

Comme le montre le peu qu'on sait de sa vie, Suchon a elle-même tenté de suivre ces prescriptions. Après avoir quitté le couvent, elle mène en effet

80. Elle se dit d'ailleurs d'accord avec Platon en ce qui concerne le gouvernement des femmes (Suchon, Autorité, p. 32).

81. Les relations avec les directeurs de conscience, comme avec les maîtres, font exception: Suchon les considère nécessaires au perfectionnement des neutralistes, mais dans la mesure où ces directeurs font eux-mêmes preuve d'une sagesse sans pareille — puisqu' «il n'appartient qu'aux sages de gouverner les autres» (Le célibat volontaire, p. 478). Il est probable que les directeurs de conscience puissent être des hommes comme des femmes. 
une vie simple et retirée; la parution de ses deux ouvrages est, peut-on croire, sa façon de se montrer utile à la société ${ }^{82}$. La condition de neutraliste, «voie du milieu » entre le mariage et le cloître ${ }^{83}$ demeure cependant un idéal vers lequel il faut tendre: à quel point Suchon l'a-t-elle réalisé, nous ne le saurons jamais. Son exemple montre néanmoins qu'elle estimait possible d'adopter cette condition même dans une société où les lois et les institutions sont profondément hostiles aux femmes. La force incroyable que ce projet démontre devrait demeurer, peut-être, notre plus grande inspiration.

\section{Bibliographie}

Angenot, Marc. Les champions des femmes: examen du discours sur la supériorité des femmes, 1400-1800, Montréal, Presses de l'Université du Québec, 1977.

Aristote (I644). La morale d'Aristote (trad. C. Catel), Toulouse, Bosc.

Bertolini, Sonia. "Gabrielle Suchon: une vie sans engagement", dans Australian Journal of French Studies 37 (3), 2000, p. 289-308.

Desnain, Véronique. "Gabrielle Suchon's Neutralists» dans Relations \& Relationships in Seventeenth-century French Literature, Tübingen: Gunter Narr Verlag, 2006, p. II6-I3I.

-. "The Origins of la vie neutre: Nicolas Caussin's Influence on the Writings of Gabrielle Suchon» dans French Studies 63 (2), 2009, p. I48-160.

—. "Gabrielle Suchon: Militant Philosophy in Seventeeth Century France» dans Forum for Modern Language Studies 49 (3), 2013, p. 257-27I.

Dorlin, Elsa. L'évidence de l'égalité des sexes: une philosophie oubliée du XVII siècle, Paris, L'Harmattan, 200I.

Dunn-Lardeau, Brenda. «La place de "La comédie des quatre femmes" de Marguerite de Navarre ( I 542) dans le discours sur le célibat volontaire comme modèle de félicité de l'Arioste à Gabrielle Suchon » dans Renaissance and Reformation 26 (4), 2002, p. II3-I39.

Fauré, Christine. La démocratie sans les femmes, Paris, Presses Universitaires de France, 1985 .

Gournay, Marie Le Jars de (I622). Égalité des Hommes et des Femmes, Paris.

Hoffman, Paul. "Le féminisme spirituel de Gabrielle Suchon» dans XVII siècle, I2I, I978, p. 269-276.

Kirsop, Wallace. "A Note on Gabrielle Suchon's Efforts to Seek Publication of her Works» dans Journal of Romance Studies 5, 2005, p. 17-1 8.

Le Doeuff, Michèle. Le sexe du savoir, Paris, Aubier, 1998.

Marinella, Lucrezia (I60I). La nobiltà et l'eccellenza delle donne: co'diffetti, et mancamenti de gli huomini, Venise, Giovanni Battista Ciotti.

Poulain de la Barre, François. De l'égalité des deux sexes; De l'éducation des dames; De l'excellence des hommes (éd. M.-F. Pellegrin), Paris, Vrin, 20 I I.

Ribard, Dinah. «Religieuses philosophes, religieuses sans clôture, ermites et vagabondes: appartenances et dissidences au XVII ${ }^{\mathrm{e}}$ siècle» dans L'Atelier $d u$ Centre de recherches historiques 4, 2009 [http://acrh.revues.org/I 367 ].

82. Suchon suggère en effet aux neutralistes éclairées qui vivraient seules d'aider leur prochain en publiant des ouvrages utiles (Suchon, Le célibat volontaire, p. 358).

83. Suchon se réclame à cet égard d'Aristote (Suchon, Le célibat volontaire, p. 6-7). 
Ronzeaud, Pierre. «La femme au pouvoir ou le monde à l'envers » dans XVII siècle, I08, I975, p. 9-33.

Schiebinger, Londa L. The Mind Has No Sex? Women in the Origins of Modern Science, Cambridge (MA), Harvard University Press, I989.

Shapiro, Lisa (à paraître). "Gabrielle Suchon's 'Neutralist': The Status of Women and the Invention of Autonomy» dans Women and Liberty: 1600-1800 (dir. Jacqueline Broad et Karen Detlefsen, Oxford, Oxford University Press, p. 220248.

Suchon, Gabrielle (1693). Traité de la morale et de la politique, Lyon: B. Vignieu. [Transcription partielle disponible au http://querelle.ca/]

- ( - ( 700$)$. Du célibat volontaire, ou la vie sans engagement, Paris, J. \& M. Guignard. 